\title{
Erratum
}

\section{Structure and Polymorphism of Class I MHC Antigen mRNA}

Ashwani K. Sood, Julian Pan, Paul A. Biro, Dennis Pereira, Rakesh Srivastava, V. B. Reddy, B. W. Duceman, and S. M. Weissman

Immunogenetics 22:101-121, 1985

The nucleotide sequence of HLA-B7 mRNA shown in Figure 2 represents an earlier "unproofed version" having incorrect nucleotide assignments at several positions. The correct sequence of HLA-B7 mRNA is as shown in Figure 3 of this paper. 